\title{
Deep learning opens new horizons in personalized medicine (Review)
}

\author{
GEORGIOS Z. PAPADAKIS ${ }^{1,2}$, APOSTOLOS H. KARANTANAS ${ }^{1,2}$, MANOLIS TSIKNAKIS ${ }^{3}$, \\ ARISTIDIS TSATSAKIS ${ }^{4}$, DEMETRIOS A. SPANDIDOS ${ }^{5}$ and KOSTAS MARIAS ${ }^{1,3}$
}

\author{
${ }^{1}$ Computational Biomedicine Laboratory (CBML), Institute of Computer Science (ICS), Foundation for Research and \\ Technology Hellas (FORTH); ${ }^{2}$ Department of Medical Imaging, Heraklion University Hospital, Medical School, \\ University of Crete, 70013 Heraklion; ${ }^{3}$ Technological Educational Institute of Crete, Department of Informatics Engineering, \\ 71410 Heraklion; ${ }^{4}$ Centre of Toxicology Science and Research, Faculty of Medicine, University of Crete, 71003 Heraklion; \\ ${ }^{5}$ Laboratory of Clinical Virology, Medical School, University of Crete, 70013 Heraklion, Greece
}

Received January 9, 2019; Accepted March 6, 2019

DOI: $10.3892 /$ br.2019.1199

\begin{abstract}
Although the idea of the personalization of patient care dates back to the time of Hippocrates, recent advances in diagnostic medical imaging and molecular medicine are gradually transforming healthcare services, by offering information and diagnostic tools enabling individualized patient management. Facilitating personalized/precision medicine requires taking into account multiple heterogenous parameters, such as sociodemographics, gene variability, environmental and lifestyle factors. Therefore, one of the most critical challenges in personalized medicine is the need to transform large, multi-modal data into decision support tools, capable of bridging the translational gap to the clinical setting. Towards these challenges, deep learning (DL) provides a novel approach, which enables obtaining or developing high-accuracy, multi-modal predictive models, that allow the implementation of the personalized medicine vision in the near future. DL is a highly effective strategy in addressing these challenges, with DL-based models leading to unprecedented results, matching or even improving state-of-the-art prediction/detection rates based on both intuitive and non-intuitive disease descriptors. These results hold promise for significant socio-economic benefits from the application of DL personalized medicine.
\end{abstract}

\section{Contents}

1. Introduction

2. Current developments

3. Conclusions and future prospects

Correspondence to: Dr Georgios Z. Papadakis, Computational Biomedicine Laboratory (CBML), Institute of Computer Science (ICS), Foundation for Research and Technology Hellas (FORTH), 100 N. Plastira Street, Vassilika Vouton, 70013 Heraklion, Greece E-mail: gzpapadakis@gmail.com

Key words: deep learning, personalized medicine

\section{Introduction}

Deep learning (DL) is a specialized machine learning approach based on multiple-layered structures (algorithms) of artificial neurons, which are able to process information and learn by adjusting the weights at each synapse, enabling the performance of an intelligent task with high precision. Furthermore, these deep networks are able to 'learn' from large amounts of data and, similar to the human brain, can generalize concepts and apply these to new data with high accuracy. This 'intelligence' property has been expanded towards deep multimodal learning by integrating different sources of information (images, text, speech etc.) for further improving high-level multimodal decision support and reasoning (1). As a result, current DL systems are able to integrate and model heterogeneous data from an individual patient, across modalities and time, allowing better predictions and recommending treatment options tailored to each patient's individual characteristics and needs (2). This concept is illustrated in Fig. 1, highlighting the potential of DL to transform large-sized healthcare data into useful tools for advancing personalized medicine. DL algorithms are well-suited for large amounts of healthcare data and perform better than traditional statistical models at the expense of sacrificing interpretability for predictive power.

\section{Current developments}

Accurate diagnosis is a milestone for personalized medicine. Ehteshami Bejnordi et al reported that 7 DL algorithms trained to detect metastases in hematoxylin- and eosin-stained tissue sections of lymph nodes of women with breast cancer, outperformed a panel of 11 pathologists with an area under the curve (AUC) of 0.994 (best algorithm) vs. 0.884 (best pathologist) (3). Madani et al presented a DL echocardiography system for the automated diagnosis of cardiac disease to address the issue of echocardiographic assessment inaccuracy (up to 30\%) of echo reports, achieving an $80 \%$ accuracy in echocardiographic view classification and a $92.3 \%$ accuracy for left ventricular hypertrophy classification (4). Recently, Ding et al proposed a DL system demonstrating an improved early prediction of the 


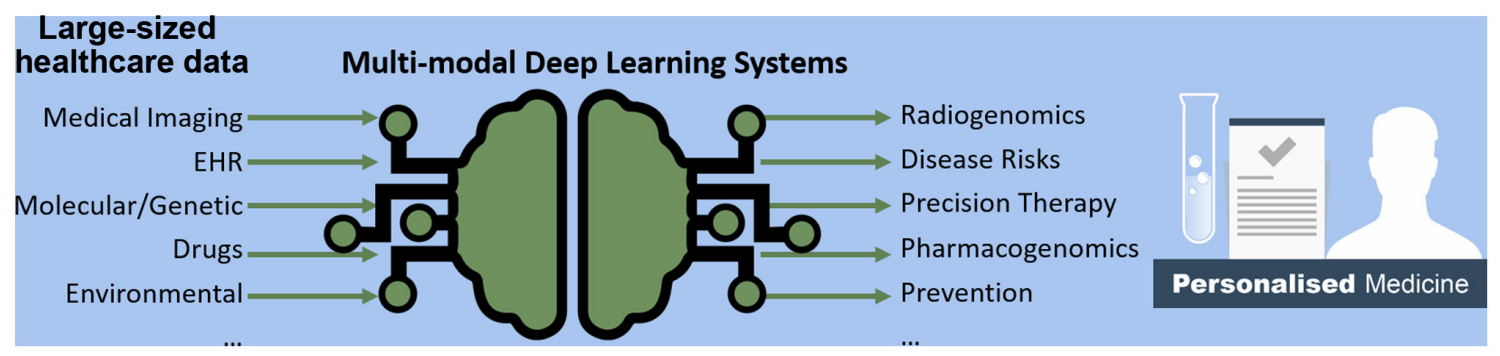

Figure 1. Multi-modal deep learning architectures can significantly contribute to the advancement of personalized medicine.

final diagnosis of Alzheimer's disease (AD) (82\% specificity at $100 \%$ sensitivity, on average of 75.8 months prior to the final diagnosis), utilizing ${ }^{18} \mathrm{~F}$-fludeoxyglucose positron emission tomography (PET) of the brain, and therefore enhancing opportunities for early therapeutic interventions (5). Finally, another recent study presented a DL algorithm (CheXNeXt) that matched the expert human radiologist performance in diagnosing 11 lung pathologies (6).

DL has begun to revolutionize decision support systems in oncology due to the plethora of the available data and the heterogeneity of various malignancies, which require algorithms able to decipher hidden phenotype and genotype patterns, as well as their associations. Recently, Causey et al reported a DL system (NoduleX) which achieved an impressive accuracy (AUC of $\sim 0.99$ ) in nodule malignancy classification ( $>1,000$ nodules) based on CT scans, matching the performance of experienced radiologists (7). Previously, Li et al reported a DL brain tumor radiogenomic system able to predict with high accuracy the IDH1 mutation status solely from multi-modal magnetic resonance imaging (MRI) data, achieving an impressive 95\% AUC (8). Using a similar approach, Akkus et al demonstrated a DL system capable of predicting the codeletion of $1 \mathrm{p} / 19 \mathrm{q}$ chromosome arms with high accuracy $(87.7 \%)$ in 159 patients with low-grade glioma (9). In both cases, DL seems to enable the discovery of hidden imaging phenotype properties, which are associated with molecular/genetic information of high prognostic value.

The in silico prediction of therapy optimization is of utmost importance for personalized medicine. Bibault et al presented a DL radiomics system capable of predicting complete response following neo-adjuvant chemoradiation for locally advanced rectal cancer with the ultimate goal of identifying patients who would benefit more from conservative treatment rather than radical resections (10). From a bioinformatics standpoint, Yousefi et al developed the SurvivalNet framework based on DL and Bayesian optimization for predicting clinical outcomes from large amounts of data, which was generated by diverse genomic platforms (11). In a recent study on 768 patients, DL outperformed the widely used Cox proportional hazard regression model as well as other widely used models for survival analysis indicating that it can enhance the role of survival prediction in individualized clinical decision making (12). Reliable health predictors based on patient's heterogeneous electronic health records (EHRs) data integration is another important application field of DL. In this direction, Miotto et al developed the 'Deep Patient' prediction system trained on 700,000 EHRs and demonstrated high disease prediction performance for severe diabetes, schizophrenia and various malignancies (13).

Drug discovery for the personalization of therapy is another area that can benefit from the flexible architecture of DL systems towards de novo molecular design, reaction and bioactivity predictions (14). In this direction, IBM and Pfizer have joined forces for advancing immuno-oncology research with Watson for Drug Discovery (www.pfizer.com/news/ press-release/press-releasedetail/ibm_and_pfizer_to_accelerate_ immuno_oncology_research_with_watson_for_drug_discovery). Moreover, predicting environmental factors that influence health status is an integral part of preventive, personalized medicine. Ong et al proposed a time-series forecasting DL system for predicting the concentration of fine particulate matter PM2.5 (interfering with both human health and climate), in Japan, using exclusively real and publicly available sensor data, outperforming currently used methods (15).

\section{Conclusions and future prospects}

DL architectures have already demonstrated significant technological advances across the personalized medicine informatics application spectrum, due to their versatility towards integrating multi-modal clinical data, discovering hidden properties and successfully generalizing results in unseen datasets. The number of DL applications for personalized medicine will continue to grow due to the high demand for such technologies and their anticipated socioeconomic impact. Although this process is facilitated by the widely available DL open computational frameworks and the abundance of large amounts of healthcare data, there is still skepticism regarding the clinical adoption of DL technologies emanating from the lack of causality and their 'black-box' nature. The inability to understand why an algorithm achieves generalization and performs so well, may be a critical factor inhibiting the clinical translation of DL technologies. To address this issue, several researchers are focusing on explaining the inner organization of DL networks. Shwartz-Ziv and Tishby, and Tishby and Zaslavsky have proposed an influential theory based on the 'information bottleneck' concept, according to which the network compresses input data as if by squeezing the information through a bottleneck, retaining only the features most relevant to the general concepts of the learning task $(16,17)$. This compression process is pronounced at the higher layers of the network for promoting better predictions via preserving information relevant to the output labels at the expense of input data information, which is gradually 'forgotten'. The confirmation of such theoretical explanatory models in conjunction 
with large-sized data validation across clinical sites and patient data registries, is a sine qua non condition for accelerating the clinical translation of DL personalized medicine decision support systems.

\section{Acknowledgements}

Not applicable.

\section{Funding}

No funding was received.

\section{Availability of data and materials}

Data sharing is not applicable to this article, as no datasets were generated or analyzed during the current study.

\section{Authors' contributions}

GZP and KM conceived and designed the study. GZP and KM researched the literature, and wrote the manuscript. AHK, MT, AT and DAS, substantially contributed to the conception and design of the study, and to the selection of the data included in the study, revised it critically for important intellectual content, and finally approved the version to be published. All authors have read and approved the final manuscript.

\section{Ethics approval and consent to participate}

Not applicable.

\section{Patient consent for publication}

Not applicable.

\section{Competing interests}

DAS is the Editor-in-Chief for the journal, but had no personal involvement in the reviewing process, or any influence in terms of adjudicating on the final decision, for this article. The other authors declare that they have no competing interests.

\section{References}

1. Noda K, Arie H, Suga Y and Ogata T: Multimodal integration learning of robot behavior using deep neural networks. Robot Auton Syst 62: 721-736, 2014.

2. Chen JH and Asch SM: Machine Learning and Prediction in Medicine - Beyond the Peak of Inflated Expectations. N Engl J Med 376: 2507-2509, 2017.
3. Ehteshami Bejnordi B, Veta M, Johannes van Diest P, van Ginneken B, Karssemeijer N, Litjens G, van der Laak JA, Hermsen M, Manson QF, Balkenhol M, et al; the CAMELYON16 Consortium: Diagnostic assessment of deep learning algorithms for detection of lymph node metastases in women with breast cancer. JAMA 318: 2199-2210, 2017.

4. Madani A, Ong JR, Tibrewal A and Mofrad MRK: Deep echocardiography: Data-efficient supervised and semi-supervised deep learning towards automated diagnosis of cardiac disease. NPJ Digit Med 1: 59, 2018.

5. Ding Y, Sohn JH, Kawczynski MG, Trivedi H, Harnish R, Jenkins NW, Lituiev D, Copeland TP, Aboian MS, Mari Aparici C, et al: A Deep learning model to predict a diagnosis of alzheimer disease by using ${ }^{18}$ F-FDG PET of the brain. Radiology 290: 456-464, 2019.

6. Rajpurkar P, Irvin J, Ball RL, Zhu K, Yang B, Mehta H, Duan T, Ding D, Bagul A, Langlotz CP, et al: Deep learning for chest radiograph diagnosis: A retrospective comparison of the CheXNeXt algorithm to practicing radiologists. PLoS Med 15: e1002686, 2018.

7. Causey JL, Zhang J, Ma S, Jiang B, Qualls JA, Politte DG, Prior F, Zhang S and Huang X: Highly accurate model for prediction of lung nodule malignancy with CT scans. Sci Rep 8: 9286, 2018.

8. Li Z, Wang Y, Yu J, Guo Y and Cao W: Deep Learning based Radiomics (DLR) and its usage in noninvasive IDH1 prediction for low grade glioma. Sci Rep 7: 5467, 2017.

9. Akkus Z, Ali I, Sedláŕ J, Agrawal JP, Parney IF, Giannini C and Erickson BJ: Predicting deletion of chromosomal arms $1 \mathrm{p} / 19 \mathrm{q}$ in low-Grade gliomas from MR images using machine intelligence. J Digit Imaging 30: 469-476, 2017.

10. Bibault JE, Giraud P, Housset M, Durdux C, Taieb J, Berger A, Coriat R, Chaussade S, Dousset B, Nordlinger B, et al: Deep Learning and Radiomics predict complete response after neo-adjuvant chemoradiation for locally advanced rectal cancer. Sci Rep 8: 12611, 2018.

11. Yousefi S, Amrollahi F, Amgad M, Dong C, Lewis JE, Song C, Gutman DA, Halani SH, Velazquez Vega JE, Brat DJ, et al: Predicting clinical outcomes from large scale cancer genomic profiles with deep survival models. Sci Rep 7: 11707, 2017.

12. Matsuo K, Purushotham S, Jiang B, Mandelbaum RS, Takiuchi T, Liu Y and Roman LD: Survival outcome prediction in cervical cancer: Cox models vs deep-learning model. Am J Obstet Gynecol: Dec 21, 2018 (Epub ahead of print), 2018. doi: 10.1016/j. ajog.2018.12.030.

13. Miotto R, Li L, Kidd BA and Dudley JT: Deep Patient: An Unsupervised representation to predict the future of patients from the electronic health records. Sci Rep 6: 26094, 2016.

14. Chen H, Engkvist O, Wang Y, Olivecrona M and Blaschke T: The rise of deep learning in drug discovery. Drug Discov Today 23: 1241-1250, 2018

15. Ong BT, Sugiura K and Zettsu K: Dynamically pre-trained deep recurrent neural networks using environmental monitoring data for predicting PM2.5. Neural Comput Appl 27: 1553-1566, 2016.

16. Shwartz-Ziv R and Tishby N: Opening the Black Box of Deep Neural Networks via Information. Intel Collaborative Research institute for Computational Intelligence (ICRI-CI). arXiv:1703.00810, 2017.

17. Tishby $\mathrm{N}$ and Zaslavsky N: Deep learning and the information bottleneck principle. In 2015 IEEE Information Theory Workshop (ITW), pp1-5, 2015. 